\title{
ON EQUIVALENT LIE FOLIATIONS
}

\author{
AMETH NDIAYE \\ Départment de Mathématiques (FASTEF) \\ Université Cheikh Anta Diop \\ Dakar \\ Senegal \\ e-mail: ameth1.ndiaye@ucad.edu.sn
}

\begin{abstract}
In this paper we study in which condition two Lie foliations are equivalent. In particular, our foliations are defined by the orbit of a group action on a compact manifold.

Every manifold in this paper is compact and our Lie group $G$ is connected and simply connected.
\end{abstract}

\section{Introduction}

Let $G$ be a simply connected Lie group and $M$ be a compact manifold of dimension $n$. A Lie $G$-foliation is the given of a set $\mathcal{F}$ of couples $(U ; f)$, where $U$ is an open subset of $M$ and $f: U \rightarrow G$ a submersion, having the following properties:

2010 Mathematics Subject Classification: Primary 53B05; Secondary 53B20.

Keywords and phrases: foliation, nilpotent, Lie group, action group, manifold, compact.

Received September 27, 2018

(C) 2019 Scientific Advances Publishers

This work is licensed under the Creative Commons Attribution International License (CC BY 3.0). http://creativecommons.org/licenses/by/3.0/deed.en_US

Open Access (c) (7) 
(i) The open sets $U$ cover $M$.

(ii) for all $(U ; f)$ and $(W ; h) \in \mathcal{F}$, there exists $g \in G$ such that, for all $x \in U \cap W$, we have $f(x)=h(x) g$.

In particular, level surfaces of the submersions $f$, for $(U ; f) \in \mathcal{F}$, recollect to form a Lie foliation on $M$. To avoid ambiguities, it is further assumed that $\mathcal{F}$ is maximal in the following sense: if $U$ is an open of $M$ and $f: U \rightarrow G$ a submersion, if, for any $(W ; h)$ in $\mathcal{F}$, there exists $g$ in $G$ with $f=h$.g on $U \cap W$, we have $(U ; f) \in \mathcal{F}$. In this case $G$ is called the transverse group of the $G$-foliation.

In the general case the structure transverse of $G$-foliation is given by Fedida's theorem [3]:

Let $\mathcal{F}$ be a $G$-foliation on a compact manifold $M$. Let $\tilde{M}$ be the universal covering of $M$ and $\widetilde{\mathcal{F}}$ the recovery of $\mathcal{F}$ on $\tilde{M}$. Then there exists a morphism $h: \pi_{1}(M) \rightarrow G$ and one locally trivial fibration $D: \tilde{M} \rightarrow G$ whose fibers are the leaves of $\tilde{\mathcal{F}}$ and such that for any $\gamma \in \pi_{1}(M)$, the following diagram is commutative:

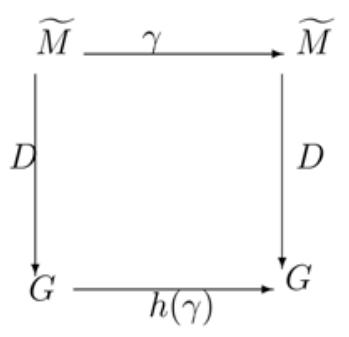

where the first line represents the transformation of $\gamma \in \pi_{1}(M)$ on $\tilde{M}$. The group $\Gamma=h\left\{\pi_{1}(M)\right\}$ (which is a subgroup of $G$ ) is called the holonomy group of $\mathcal{F}$ and the fibration $D: \tilde{M} \rightarrow G$ is called the developing application of the foliation $\mathcal{F}$. This structure theorem makes it possible to build Lie foliation on a manifold. 
If $\mathbf{g}$ is the Lie algebra of $G$. A Maurer-Cartan form with values in $\mathbf{g}$ is a differential 1-form $\omega \in \Omega^{1}(M, \mathbf{g})$ with trivial formal, i.e.,

$$
d \omega+\frac{1}{2}[\omega, \omega]=0 .
$$

Here $[\omega, \omega]$ is the differential 2 -form on $M$ with values in $\mathbf{g}$ given by $[\omega, \omega](X, Y)=[\omega(X), \omega(Y)]$. If $\omega$ is non singular, i.e., if $\omega_{x}: T_{x}(M) \rightarrow \mathbf{g}$ is surjective at any point $x \in M$, then the dimension of $\mathbf{g}$ is finite and $\operatorname{ker}(\omega)$ is subbundle of $T(M)$ of codimension dim g. Vanishing of the formal curvature implies that the subbundle $\operatorname{ker}(\omega)$ is involutive and hence defines a foliation $\mathcal{F}$ on $M$, with $\operatorname{codim} \mathcal{F}=\operatorname{dim} \mathbf{g}$ and

$$
T(\mathcal{F})=\operatorname{ker}(\omega)
$$

A Lie foliation is a foliation defined in this way by a non-singular Maurer-Cartan form. Lie $G$-foliations form a very special class of foliations and satisfy various strong properties. For example, any left invariant Riemannian metric of $G$ gives rise to a metric on the normal bundle of $\mathcal{F}$, invariant by the holonomy pseudogroup; that is, $\mathcal{F}$ is a Riemannian foliation. Each leaf of $\mathcal{F}$ has trivial holonomy and they are mutually Lipshitz diffeomorphic. Conversely by the work of Molino [14], the study of Riemannian foliations reduces to that of Lie foliations. See [15] for detailed accounts. Classical examples of Lie $G$ foliations are:

Example 1.1. Let $H$ be a Lie group and a surjective homomorphism $f: H \rightarrow G$, and let $\Gamma$ be a uniform lattice of $H$. Then the right action of $N=\operatorname{ker}(f)$ on the quotient space $H / \Gamma$, gives rise to a Lie $G$-foliation, with leaves diffeomorphic to $N / \Gamma \cap N$.

Assume $G$ is compact, let $B$ be a closed manifold, and let $\phi: \pi_{1}(B) \rightarrow G$ be a group homomorphism. The suspension gives a fibration $G \rightarrow M \rightarrow B$ together with a Lie $G$-foliations transverse to the fibers; the leaf is the ker $\phi$ covering of $B$. 
We study in this paper when two Lie foliations are topologically equivalent, or, more generally, whether there is a continuous map $f$ from $M_{1}$ to $M_{2}$ whose restriction to each leaf of $\mathcal{F}_{1}$ is a covering map onto a leaf of $\mathcal{F}_{2}$ (with $\mathcal{F}_{i}$ Lie foliation of $M_{i}, i=1,2$ ). If the foliations are obtain by an action, Benardete studied the question and gave some results.

\section{Homogeneous Foliations}

An automorphism of a foliated manifold $(M, \mathcal{F})$ is a diffeomorphism $\phi: M \rightarrow M$ which preserves the foliation, i.e., for which the image of any leaf is a leaf, or equivalently, $d \phi(T(\mathcal{F}))=T(\mathcal{F})$. The automorphisms of $(M, \mathcal{F})$ form a group which we shall denote by $\operatorname{Aut}(M, \mathcal{F})$.

A foliation $\mathcal{F}$ of a manifold $M$ is called homogeneous if the group $\operatorname{Aut}(M, \mathcal{F})$ acts transitively on $M$, or in other words, if for any two points $x, y \in M$, there exists a diffeomorphism $\phi: M \rightarrow M$ which preserves the foliation and maps $x$ to $y$.

Example 2.1. Let $\mathcal{F}$ be a foliation of a manifold $M$ and let $x$ and $y$ be two points of $M$ lying on the same leaf of $\mathcal{F}$. Then $\mathcal{F}$ is a homogeneous foliation of $M$.

In particular, all the leaves of a homogeneous foliation are diffeomorphic. In this following example we have a homogeneous foliation.

Example 2.2. Let $M$ be a differential manifold dimension $n+m$ and $G$ be a connected Lie group of dimension $m$. An action of $G$ on $M$ is the $\operatorname{map} \phi: G \times M \rightarrow M$ given by

(1) $\phi(e, x)=x$ for all $x \in M$ (where $e$ is unit element of $G$ ).

(2) $\phi\left(g^{\prime}, \phi(g, x)\right)=\phi\left(g g^{\prime}, x\right)$, for all $x \in M$ and $g, g^{\prime} \in G$. 
Suppose for all $x \in M$, the dimension of the isotropy group $G_{x}=\{g \in G: \phi(g, x)=x\}$ is not depend to $x$. Then the action $\phi$ define a foliation $\mathcal{F}$ of dimension $m-\operatorname{dim} G_{x}$; these leaves are the orbits $\{\phi(g, x): g \in G\}$. In particular, it is the case where the isotropy group is discret. As an integrable subbundle of $T(M)$, this foliation can simply be described in terms of Lie algebra $\mathbf{g}$ of $G$, namely, as the image of derivative of the action, which is a map of vector bundles $\mathrm{g} \times M \rightarrow T(M)$ of constant rank.

In the case where $M=H / \Gamma$ with $H$ a Lie group, $\Gamma$ a discret subgroup and $G$ a connected Lie subgroup of $H$; then the foliation $\mathcal{F}$ obtained by the action of $G$ on $M$ is a homogeneous foliation.

Proposition 2.3. The homogeneous foliation thus defined is a Lie foliation whose transverse group is $G$.

Proof. Let $H$ be a simply connected Lie group, $\Gamma$ be a $H$ lattice and $G$ be a connected Lie subgroup of $H$. By definition the action of $G$ on the manifold $H / \Gamma$ defines a $\mathcal{F}$ which is homogeneous on $H / \Gamma$.

Since the map $\rho: H \rightarrow G$ is a surjective morphism, then $\mathcal{F}$ is a Lie foliation whose transverse group is $G$. In fact the images by $\rho$ of the classes $H$ modulo ker $\rho$ are the $G$-foliation on $H / \Gamma$ which coincides with $\mathcal{F}$. Its developing map is $\rho$ and its holonomy group is $\Gamma$.

Example 2.4. Let $G$ be a simply connected Lie group, $H$ be a connected subgroup of $G$ and $\Gamma$ be a lattice in $G$. The orbits of $H$ define a foliation $\mathcal{F}$ on $G / \Gamma$ which is a homogeneous Lie $G$-foliation.

Let $\mathcal{F}_{1}$ and $\mathcal{F}_{2}$ defined respectively by the action of $G_{1}$ on the manifold $M_{1}=H_{1} / \Gamma_{1}$ and the action of $G_{2}$ on $M_{2}=H_{2} / \Gamma_{2}$. If $H_{i}$ is a simply connected solvable Lie group, $\Gamma$ a lattice of $H_{i}$ and $G_{i}$ a connected Lie subgroup of $H_{i}$, then Benardete-Witte have showed 
Theorem 2.5 ([9]). If the maximal tore of $H_{i}$ is include in $\overline{\operatorname{Ad}_{H_{i}} \Gamma_{i}}$, then $\mathcal{F}_{1}$ is topologically equivalent to $\mathcal{F}_{2}$ there exist a isomorphism $\sigma: H_{1} \rightarrow H_{2}$ such that $\sigma\left(G_{1}\right)=G_{2}$ and $\sigma\left(\Gamma_{1}\right)$ is conjugate to $\Gamma_{2}$.

We want to generalisate this result for any two nilpotent Lie foliation.

\section{Equivalence of Nilpotents Lie Foliations}

The nilpotent Lie foliations has been classified in inverse image by Haefliger. The following theorem characterizes the holonomy groups of such foliations. In this section, we recall some results of rigidity of lattices before stating the equivalence theorem of Lie foliations.

Proposition 3.1 ([11]). Let $G_{1}$ and $G_{2}$ be two simply connected nilpotent Lie groups, and $K_{1}$ be a uniform subgroup of $G_{1}$ : then any continuous homomorphism $h: K_{1} \rightarrow G_{2}$ extends to a unik continuous homomorphism $\bar{h}: G_{1} \rightarrow G_{2}$.

Proposition 3.2 ([11]). Let $\Gamma$ be a nilpotent Lie group, of finite type, and without torsion. Then there exist a simply connected nilpotent Lie group $\widetilde{G}$ and an injective homomorphism $J: \Gamma \rightarrow \widetilde{G}$ such that $J(\Gamma)$ is a discrete uniform subgroup $\widetilde{G}$.

Theorem 3.3 ([6]). In a nilpotent Lie group G any finite type uniform subgroup is feasible as a holonomy group of Lie G-foliation on a compact manifold.

It is shown that a Lie foliation defined by the Lie group $\mathbb{R}^{n}$ is close to a foliation with closed leaves and the compact support is fiber on $\mathbb{R}^{n} / \Gamma$, where $\Gamma$ is a of $\mathbb{R}^{n}$ : For this, we deform the holonomy group $\Gamma$ to get a discrete subgroup of $\mathbb{R}^{n}$. The reciprocal classification is known thanks to the work of Mal'tsev and Haefliger, more precisely we have 
Theorem 3.4 ([6]). Any nilpotent Lie foliation on a compact manifold is a reverse image of a homogeneous foliation.

Using the results of Haefliger and Benardete we show that

Theorem 3.5. Let $\mathcal{F}_{1}$ and $\mathcal{F}_{1}$ be two nilpotent Lie foliations on a compact manifold whose respective transverse groups are $G_{1}$ and $G_{2}$. Then $\mathcal{F}_{1}$ and $\mathcal{F}_{1}$ are topologically equivalent.

Proof. Let $\Gamma_{i}$ be the holonomy group of the nilpotent Lie $G_{i}$-foliation $\mathcal{F}_{i}$ (with $i=1,2$ ), $\Gamma_{i}$ is a uniform subgroup and finite type of $G_{i}$. We know, according to [11], $\Gamma_{i}$ is a cocompact lattice in a nilpotent Lie group $H_{i}$, the canonical injection $\rho: \Gamma_{i} \rightarrow G_{i}$ extend to a continous homomorphism $\rho^{\prime}: H_{i} \rightarrow G_{i}$ so we have $\rho=\rho^{\prime} \circ j$, where $j: \Gamma_{i} \rightarrow H_{i}$. We obtain an exact small sequence

$$
1 \rightarrow F_{i} \rightarrow H_{i} \rightarrow G_{i} \rightarrow 1,
$$

which define a homogeneous Lie $G_{i}$-foliation on the manifold $H_{i} / \Gamma_{i}$. We call $\mathcal{F}_{i}^{\prime}$ this foliation.

Since the foliations $\mathcal{F}_{1}^{\prime}$ and $\mathcal{F}_{2}^{\prime}$ are homogeneous then they are defined by the action of $G_{i}$ on $H_{i} / \Gamma_{i}$. So since [9], $\mathcal{F}_{1}^{\prime}$ is topologically equivalence to $\mathcal{F}_{2}^{\prime}$.

$\mathcal{F}_{1}^{\prime}$ is inverse image of $\mathcal{F}_{1}$ and $\mathcal{F}_{2}^{\prime}$ is inverse image of $\mathcal{F}_{2}$, hence the result.

Remark 3.6. In the case where our transverses groups are solvable and not nilpotent the theorem may not work because Meigniez has shown that there are solvable Lie foliation which are not the inverse image of any homogeneous Lie foliation. 
AMETH NDIAYE

\section{References}

[1] D. Tischler, On fibering certain foliated manifold over $S^{1}$, Topology 9(2) (1970), 153-154.

DOI: https://doi.org/10.1016/0040-9383(70)90037-6

[2] E. Fedida, Feuilletages du plan, feuilletage de Lie, Thése Université Louis Pasteur, Strasbourg, Lecture Notes in Mathematics 652 (1973), 183-195.

[3] E. Fedida, Sur les feuilletages de Lie, C. R. Acad. Sci. Paris 272 (1971), 999-1002.

[4] S. Riche, Sur les représentations des groupes algébriques et des groupes quantiques.

[5] A. I. Mal'tsev, On a class of homogeneous spaces of nilpotent Lie groups, Izv. Akad. Nouk. SSR Ser. Mat. 13 p. 9 AMS Trans. 1-39.

[6] A. Haefliger, Groupoides d'holonomie et classifiants: Structures transverses des feuilletages, Toulouse 1982, Astéristique 116, SMF, 1984.

[7] G. Meigniez, Feuilletages de Lie résolubles, Annales de la Faculté des Sciences de Toulouse 4(4) (1995), 801-817.

DOI: https://doi.org/10.5802/afst.812

[8] H. Dathe and A. Ndiaye, Sur les feuilletages de Lie homogènes, Journal des Sciences et Technologies 10(1) (2011), 42-47.

[9] D. Benardete, Topological equivalence of flows on homogeneous spaces, and divergence of one-parameter subgroups of Lie groups, Trans. Amer. Math. Soc. 306(2) (1988), 499-527.

DOI: https://doi.org/10.1090/S0002-9947-1988-0933304-3

[10] D. Benardete and S. G. Dani, Topological equivalence and rigidity of flows on certain solvmanifolds, Ergodic Theory and Dynamical Systems 19(3) (1999), 559-569.

DOI: http://dx.doi.org/10.1017/S0143385799130116

[11] M. S. Raghunathan, Discrete Subgroups of Lie Groups, Springer-Verlag, New York, 1972.

[12] D. Witte, Topological equivalence of foliations of homogeneous spaces, Trans. Amer. Math. Soc. 317(1) (1990), 143-166.

DOI: https://doi.org/10.1090/S0002-9947-1990-0942428-5

[13] D. Witte, Superrigidity of lattices in solvable Lie groups, Inventiones Mathematicae 122(1) (1995), 147-193.

DOI: https://doi.org/10.1007/BF01231442 
[14] P. Molino, Géométrie globale des feuilletages riemanniens, Indagationes Mathematicae (Proceedings) 85(1) (1982), 45-76.

DOI: https://doi.org/10.1016/1385-7258(82)90007-5

[15] P. Molino, Riemannian Foliations, Birkhauser, 1988.

[16] A. S. Diallo and A. Ndiaye, On completely solvable Lie foliation, Global Journal of Advanced Research on Classical and Modern Geometries 7(2) (2018), 102-106. 